\title{
EXTRAÇÃO DO PARACETAMOL E ANÁLISE DA DISPERSÃO DA MASSA DE COMPRIMIDOS: COMPARANDO MEDICAMENTOS GENÉRICO E DE REFERÊNCIA EM LABORATÓRIO DE ENSINO
}

\author{
Lourenço Luis Botelho de Santana ${ }^{\mathrm{a}, \mathrm{b}, \#}$, Orlando Maia Barbozab, Fernando Alves Barretto ${ }^{\mathrm{a}}$, Wilson Araujo Lopes ${ }^{\mathrm{a}, \mathrm{c}}$ e Silvio $^{\mathrm{b}}$ \\ Cunha ${ }^{a, c, *,(i)}$ \\ anstituto de Química, Universidade Federal da Bahia, 40170-115 Salvador - BA, Brasil \\ bepartamento de Ciências da Vida, Universidade do Estado da Bahia, 41195-000 Salvador - BA, Brasil \\ 'INCT de Energia e Ambiente, Universidade Federal da Bahia, 40170-290 Salvador - BA, Brasil
}

Recebido em 30/03/2020; aceito em 15/06/2020; publicado na web em 23/07/2020

\begin{abstract}
EXTRACTION OF PARACETAMOL AND MASS DISPERSION ANALYZES OF TABLETS: COMPARING GENERIC AND BRAND NAME DRUGS IN UNDERGRADUATE EXPERIMENT. Medicines are employed in undergraduate courses as motivational strategy. The experiment herein developed compares the extraction of paracetamol from generic and brand name drugs. Tablets were used from two commercial sources purchased from local drugstores. Twenty tablets of each drug type were individually weighed to average weight analysis and mass dispersion was statistically analyzed in simulating of quality control. Average weight had variation in accordance with the limit of $\pm 5 \%$ specified by Brazilian Pharmacopeia. Tablets of each drug were pulverized and extracted in triplicate with ethyl acetate, and the active ingredient was obtained and identified by melting point and FTIR analyzes. Comparing different student groups at different times, there was change in the mass of paracetamol extracted by a simple extraction, affording mass recovery in the range of $59 \pm 17 \%$ to $28 \pm 1 \%$. However, mass recovery of paracetamol extraction of generic and brand name drugs was similar in each student group. Under Soxhlet extraction condition mass recovery was $80-85 \%$. The procedure developed was adequate to undergraduate laboratory, involves simple infrastructure and execution, tablets are easily obtained, and allows discussion on pharmaceutical equivalence and interchangeability.
\end{abstract}

Keywords: acetaminophen; laboratory instruction; drugs; pharmaceuticals.

\section{INTRODUÇÃO}

Medicamentos sempre chamam a atenção da população. No momento que escrevemos este artigo o mundo enfrenta a pandemia da COVID-19 e alguns fármacos estão em evidência no noticiário, como cloroquina, ibuprofeno e paracetamol. Empregar assuntos do cotidiano em aulas experimentais de química orgânica aproxima o treinamento de laboratório à realidade dos estudantes e motiva a adesão ao curso por mostrar uma visão mais prática da ciência e a importância do domínio da técnica na atuação do futuro profissional. ${ }^{1}$ Não por acaso, fármacos e medicamentos são temas recorrentes em aulas experimentais de química, e alguns estão apresentados na Figura 1 constituindo uma espécie de "prateleira pedagógica" na qual aspectos variados são explorados, tais como síntese do princípio farmacologicamente ativo (PFA), análise qualitativa e quantitativa, transformações controladas, extração e propriedades de acidez-basicidade. ${ }^{2}$ Surpreendentemente, medicamentos genéricos são raramente explorados nessas aulas, ou quando são isso não é claramente explicitado. Mais importante, não há relato de experimento que os compare aos medicamentos de referência.

Recentemente, um caso de fake news genuinamente brasileira, episódio de curandeirismo travestido de ciência, o escândalo da fosfoetanolamina trouxe à tona, entre outras coisas, a total falta de controle de qualidade na produção das cápsulas, inclusive com grande variação na massa. ${ }^{3} \mathrm{O}$ controle da dispersão da massa de medicamentos sólidos é um dos itens das boas práticas de fabricação, regulamentada pele Agência Nacional de Vigilância Sanitária (ANVISA). ${ }^{4}$ A investigação de uma variável tão elementar como a massa dos comprimidos de medicamentos não tem sido explorada

*e-mail: silviodc@ufba.br

\#e-mail alternativo: 1lsantana@uneb.br

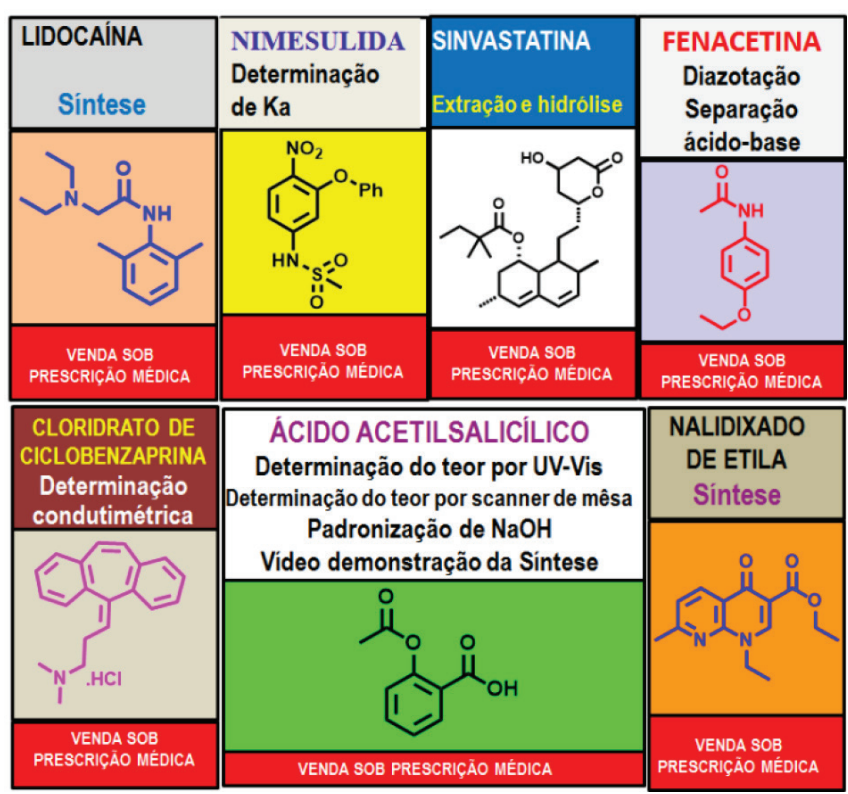

Figura 1. Prateleira pedagógica: exemplos de contextos de medicamentos em aulas experimentais ${ }^{2}$

em aula experimental de química orgânica, a despeito da aferição de massa ser uma atividade rotineira entre profissionais da química e da farmácia.

Paracetamol, $\mathrm{N}$-(4-hidroxifenil)acetamida ou acetaminofeno é o PFA presente em medicamentos genéricos e de referência, predominando o emprego do de referência em aulas práticas em diversos contextos; o genérico foi usado apenas por Araújo e colaboradores, Figura $2 \cdot{ }^{5-7}$ A extração do paracetamol de comprimidos de medicamento de 
referência é descrita em dois excelentes experimentos de graduação. No primeiro, que é uma proposta de sequência planejada de reações químicas para curso experimental avançado, faz-se a clássica extração sólido-líquido, contudo, o objetivo principal é a preparação do PFA para a síntese da dulcina. ${ }^{5}$ No segundo, em que o objetivo é explorar a acidez relativa de uma mistura de substâncias orgânicas, o paracetamol é extraído com solvente reativo (solução de $\mathrm{NaOH}$ ) de medicamento composto por ele, ácido acetilsalicílico e cafeína. Todavia, a recuperação do paracetamol é muito baixa (máximo de $20 \%$ de massa recuperada). ${ }^{6}$

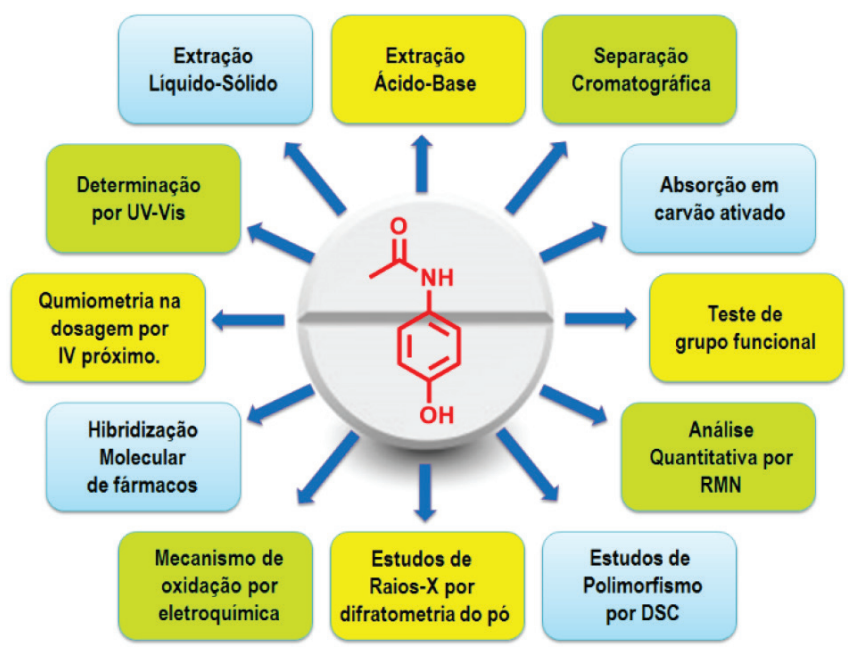

Figura 2. Contextos de aulas experimentais empregando comprimido de paracetamol $^{5-7}$

Temos continuado interesse no desenvolvimento de experimentos que contemplem aspectos motivacionais e que sejam exequíveis em laboratórios de ensino com infraestrutura simples, mas que proporcionem a investigação dos princípios químicos e a rigorosa formação profissional. ${ }^{8}$ A utilização de medicamentos contextualiza a aula prática e é uma alternativa em cenário de dificuldade de aquisição de reagentes. Consideramos, também, a facilidade de execução com procedimentos adequados ao tempo de aula, que consigam envolver o estudante em discussão sobre temas relevantes para sua futura profissão. Dessa forma, o paracetamol foi aqui revisitado pois não há relato de comparação entre a sua extração de medicamentos genéricos e de referência em aula experimental de química orgânica, nem a avaliação da dispersão das massas dos comprimidos, simulando o controle de qualidade de medicamento sólido. $\mathrm{O}$ experimento aqui desenvolvido tem como proposta sanar essas lacunas.

Para auxiliar o estudante a compreender o contexto da comparação entre os dois tipos de medicamentos é importante discorrer, mesmo que de forma breve, sobre as semelhanças e diferenças entre medicamentos genéricos e de referência.

\section{Aspectos gerais dos medicamentos de referência e genérico}

A produção de medicamentos genéricos foi iniciada nos Estados Unidos na década de 1960, sendo o pioneiro na adoção de uma legislação sobre esses medicamentos. Posteriormente, Canadá, Alemanha, Japão, Grã-Bretanha, Dinamarca e Holanda, entre outros, adotaram a mesma política. ${ }^{9}$ No Brasil, em 1976, as indústrias farmacêuticas foram autorizadas a produzir e comercializar medicamentos idênticos aos de referência. ${ }^{10}$ A legislação brasileira permitia que as próprias empresas definissem qual seria o medicamento de referência para o registro de seus produtos alternativos. Entretanto, não havia exigência de comprovação da equivalência terapêutica do medicamento alternativo em relação ao produto considerado como referência. ${ }^{10}$ Era também permitido o registro de formas farmacêuticas e dosagens diferentes em relação ao medicamento de referência havendo, ainda, casos de formulações distintas, o que deu origem ao registro de muitas especialidades farmacêuticas contendo o mesmo fármaco, vendidas com diversos nomes comerciais por diferentes laboratórios. ${ }^{10,11}$

Para se contrapor a este cenário, a Presidência da República publicou legislação que passou a ser conhecido como a Lei dos Genéricos $^{12}$ como parte da Política Nacional de Medicamentos, voltada ao estímulo da concorrência e, por consequência, da maior variedade de oferta desses produtos no mercado, e para melhorar a qualidade de todos os medicamentos, reduzir preços e, especialmente, facilitar o acesso da população aos tratamentos medicamentosos..$^{12,13}$

Toda nomenclatura empregada atualmente foi definida nesta Lei, que estabeleceu as definições de medicamentos de referência e genéricos. O medicamento de referência é um produto inovador, detentor de patente registrada no órgão federal responsável pela vigilância sanitária, com eficácia e segurança comprovada durante o seu desenvolvimento, através de ensaios clínicos, como o estudo de biodisponibilidade, realizados previamente à solicitação de registro para comercialização. ${ }^{12} \mathrm{Um}$ exemplo de produto de referência é o medicamento analgésico e antitérmico Tylenol ${ }^{\circledR}$ que apresenta o paracetamol como PFA. Já os genéricos são medicamentos que apresentam os mesmos princípios ativos, nas mesmas concentrações, indicações terapêuticas, posologia, forma farmacêutica e via de administração que o medicamento de referência, podendo diferir em características como embalagem, excipientes e veículos, tamanho, forma do produto e prazo de validade. São intercambiáveis aos de referência, e devem ser submetidos aos mesmos testes de qualidade, eficácia e bioequivalência (mesma biodisponibilidade), Figura 3. ${ }^{12}$

Os medicamentos genéricos não apresentam nome comercial e são divulgados a partir da Denominação Comum Brasileira (DCB) do(s) princípio ativo(s) ou, na ausência dela, pela Denominação Comum Internacional (DCI). Por exemplo, o medicamento genérico do Tylenol ${ }^{\circledast}$ é denominado Paracetamol, sendo obrigatório uma embalagem diferenciada contendo uma tarja amarela com a letra "G" em destaque para fácil identificação, Figura $3 .{ }^{14}$ Os medicamentos genéricos somente podem ser produzidos após o fim de uma proteção por patente ou de outro direito de exclusividade do medicamento de referência e, segundo a Resolução da ANVISA RDC n ${ }^{\circ} 135$, de $2003,{ }^{15}$ os genéricos devem ser submetidos e aprovados em testes com o intuito de comprovar que o medicamento a ser disponibilizado tenha todas as propriedades do medicamento de referência para que possam ser de fato intercambiáveis..$^{12,13}$
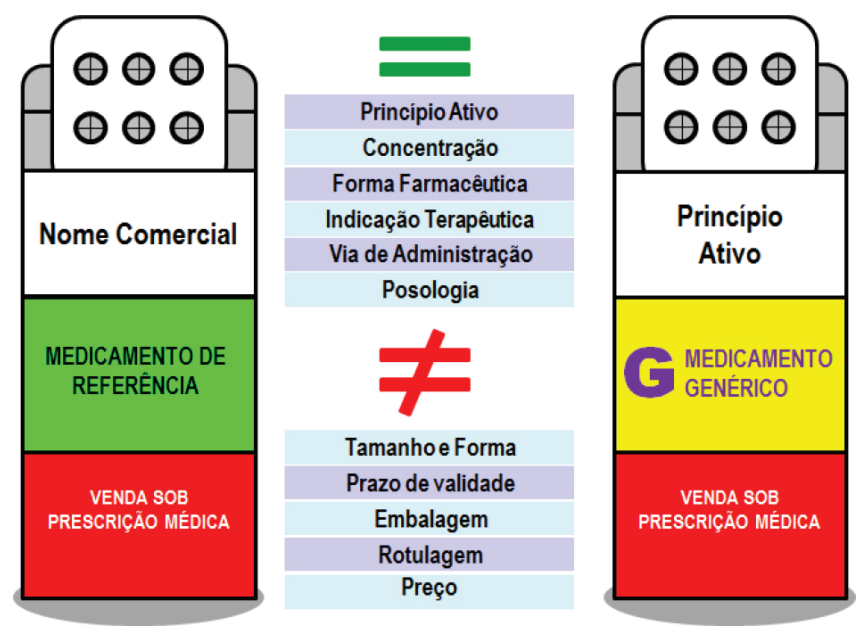

Figura 3. Igualdades e diferenças entre medicamentos genéricos e de referência 
A intercambialidade entre o genérico e seu respectivo medicamento de referência corresponde à substituição de produtos com a mesma qualidade, eficácia e segurança, sem comprometer o sucesso do tratamento, e se baseia no conceito de equivalência farmacêutica entre eles, geralmente assegurado pela comprovação da equivalência terapêutica, da bioequivalência de acordo com a Resolução da ANVISA n ${ }^{\circ} 135$ e das Boas Práticas de Fabricação de Medicamentos definidas pela Resolução da ANVISA no 301 , de $2019 .{ }^{16}$ A equivalência farmacêutica entre dois medicamentos relaciona-se à comprovação de que ambos contém o mesmo fármaco (mesma base, sal ou éster da mesma molécula terapeuticamente ativa) na mesma dosagem e forma farmacêutica, o que pode ser avaliado por meio de metodologias analíticas e testes in vitro. ${ }^{17}$ A determinação da bioequivalência consiste na demonstração de equivalência farmacêutica entre produtos apresentados sob a mesma forma farmacêutica, contendo idêntica composição de princípios ativos e que tenham comparável biodisponibilidade. ${ }^{18}$

A biodisponibilidade indica a velocidade e a extensão de absorção de um princípio ativo em uma forma de dosagem, a partir de sua curva concentração/tempo na circulação sistêmica ou sua excreção na urina; medicamentos bioequivalentes são equivalentes farmacêuticos que, ao serem administrados na mesma dose molar e condições experimentais, não apresentam diferenças significativas em relação à biodisponibilidade..$^{12,13}$ No contexto da biodisponibilidade e da bioequivalência, as formas farmacêuticas sólidas, em que insere-se o exemplo do paracetamol empregado neste experimento, merecem especial atenção, uma vez que sua dissolução pode ser afetada significativamente pelas características inerentes ao próprio fármaco, bem como pela presença de excipientes que favorecem ou dificultam a dissolução, além das técnicas de fabricação empregadas. ${ }^{19}$

Pelo fato do medicamento genérico utilizar o produto de referência como base para sua fabricação, não há gastos em pesquisas e publicidades proporcionando, na maioria das vezes, a comercialização com preços menores, até $35,5 \%$ mais baratos, com crescente participação no mercado brasileiro, tornando-se uma alternativa aos medicamentos de referências, os quais vários são importados e caros. ${ }^{13}$

\section{Estratégia da aula experimental}

A aula experimental em tela é composta por dois ciclos, sendo que, no primeiro, são disponibilizadas caixas dos medicamentos genérico e de referência de forma que cada aluno/equipe tenha acesso às duas categorias de medicamentos $(750 \mathrm{mg}$ e/ou $500 \mathrm{mg}$ em cada caso). No segundo ciclo é executada a extração do PFA dos comprimidos de cada medicamento, Figura 4. Todos os medicamentos foram adquiridos em farmácias locais e o número total de caixas é proporcional à dimensão da turma, pois cada aluno/equipe extrai 1,5 gramas de medicamento, podendo fazer em duplicata ou triplicata, dependendo do tamanho da turma e da duração da aula. Essa decisão requer o planejamento do experimento por parte do professor e está vinculada à infraestrutura do laboratório de ensino.

Por ser de rápida execução, o primeiro ciclo pode ser associado a uma etapa de instrução de laboratório (pré-lab) e o segundo pode ser executado de forma independente numa aula seguinte, principalmente quando a duração da aula é de duas horas. Para durações superiores, os dois ciclos podem ser executados num só dia. No segundo ciclo, os alunos executam esta etapa sozinhos ou em duplas, de maneira que cada aluno/equipe efetue ao menos uma extração de cada tipo de medicamento, sendo que o mínimo de três extrações do genérico e três do medicamento de referência são realizadas, para compor um quadro com triplicadas. Outros arranjos são possíveis em função do número de estudantes na turma e da infraestrutura laboratorial, mas descrevemos aqui apenas o cenário minimalista para ainda assim garantir a efetividade do tratamento estatístico e a consequente comparação, como discutido nas próximas seções.

A aula foi conduzida em três grupos de estudantes; um do curso de Química da Universidade Federal da Bahia (UFBA), em 2009, e dois do curso de Farmácia da Universidade do Estado da Bahia (UNEB), em 2018 e 2019. Para comparar os desempenhos entre profissionais em formação e profissionais experientes, os dois ciclos foram também realizados pelos autores do artigo com as amostras de 2018.

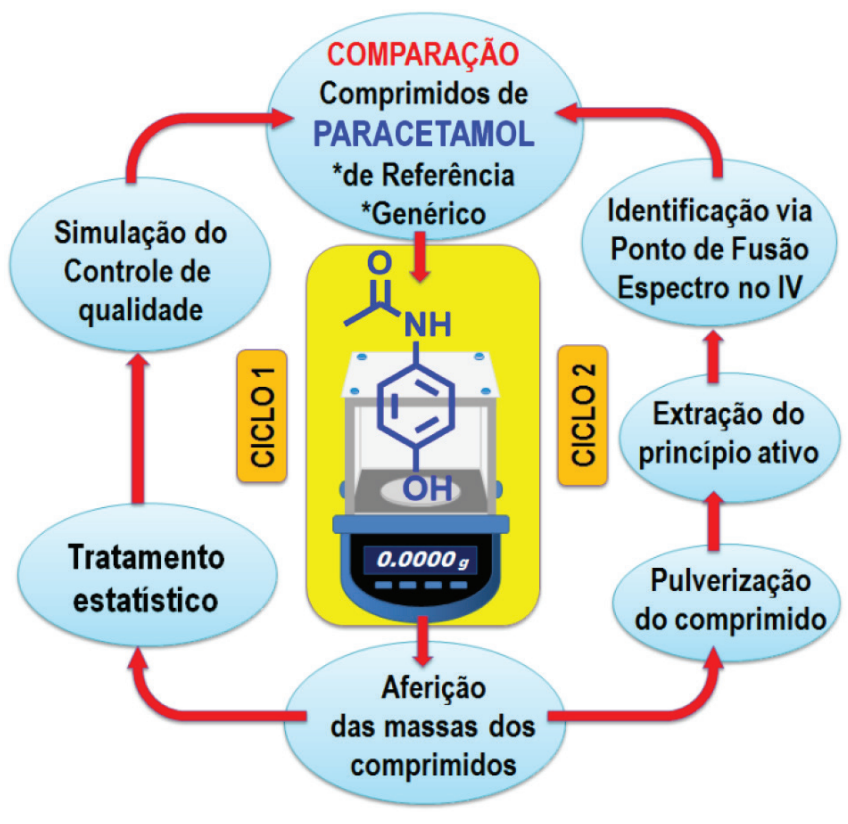

Figura 4. Estratégia da aula experimental de comparação dos medicamentos genérico e de referência contendo paracetamol

\section{Aferição da massa dos comprimidos de referência e genérico}

No primeiro ciclo do experimento são disponibilizados comprimidos contendo paracetamol de medicamentos de referência $(750 \mathrm{mg}$ e/ou $500 \mathrm{mg}$ ) e genérico (750 e/ou $500 \mathrm{mg}$ ) de acordo com a estratégia definida pelo instrutor. A quantidade de comprimidos necessária para o segundo ciclo depende do número de alunos/equipes da turma, mas neste primeiro ciclo apenas vinte comprimidos de cada tipo de medicamento são aferidos, simulando o controle de qualidade indicado na Farmacopeia Brasileira. ${ }^{4}$

Nessa etapa, é importante chamar a atenção para não tocar nos comprimidos, a despeito de, no uso cotidiano, fazermos isso quando ingerimos um comprimido, mas aqui o comprimido é tratado como um reagente químico, portanto, não deve ser tocado e sua manipulação é realizada com pinça metálica ou espátula durante todo o experimento. Adicionalmente, nesse primeiro ciclo é feita a aferição da massa de cada comprimido com balança analítica ou semianalítica, e tocar altera essa medida. Na distribuição dos comprimidos deve-se garantir que cada aluno/equipe pese ao menos um comprimido de cada tipo e registre o valor medido e seu nome no quadro do laboratório, em coluna previamente designada como massa do genérico e do de referência, para que todos possam saber o valor individual aferido. Alternativamente, a turma pode eleger dois estudantes para essa tarefa, um para pesar os comprimidos do genérico e outro para o de referência, e as duas estratégias foram testadas, sendo que recomendamos a primeira pois envolve muito mais a participação de todos na atividade.

A Tabela 1 reúne os dados coletados por grupos diferentes de estudantes perfazendo o total de seis amostras, e uma análise realizada por profissionais para fins de comparação. A primeira coisa que salta 
à vista dos estudantes é o fato da massa de todos os comprimidos ser maior que o valor nominal indicado para o PFA, o que suscita a discussão sobre a composição de medicamentos, que não é restrita apenas ao fármaco e contempla outros constituintes.

Outro aspecto que impressiona os iniciantes é a variação de massa apresentada para comprimidos de uma mesma caixa de medicamentos. Nesse ponto, o tratamento estatístico dos dados proporciona a comparação entre os dois tipos de medicamentos. A partir dos valores obtidos para as massas individuais encontradas foram calculados mediana, média, desvio médio e desvio padrão para cada caixa, Tabela 1. Adicionalmente, como são comprimidos acima de $250 \mathrm{mg}$, foram calculados os valores da média $\pm 5 \%$ para avaliar a tolerância na flutuação das massas, como especificado pela Farmacopeia Brasileira, que determina que "pode-se tolerar não mais que duas unidades fora dos limites de $\pm 5 \%$, em relação ao peso médio, porém, nenhuma poderá estar acima ou abaixo do dobro das porcentagens indicadas". ${ }^{4}$

O tratamento estatístico das medidas das massas realizadas por diferentes grupos de alunos em anos diferentes, e por profissionais experientes, indica que todas as caixas/lotes dos dois tipos de medicamentos avaliados se enquadram dentro do controle de qualidade preconizado para este item, Tabela 1. A variação observada das massas máxima e mínima permitidas para cada conjunto de comprimido está muito inferior aos limites permitidos do valor da média $\pm 5 \%,{ }^{4} \mathrm{e}$ isso é refletido nas medidas de dispersão em relação à média de cada caixa de comprimido avaliada. Assim é que, mesmo tomado o valor da média \pm desvio padrão, que apresenta a dispersão na mesma unidade da média (mg) e, nesse caso, a dispersão aferida foi a maior de todas, ainda assim a dispersão é muito pequena quando comparado a cada valor médio. Em adição a isso, o valor da mediana de cada grupo de medida é muito próximo do valor médio, reforçando a pequena dispersão das massas aferidas. Portanto, segundo todos os parâmetros calculados, os dois tipos de comprimidos são comparáveis quanto ao atendimento ao controle de qualidade da dispersão das massas dos comprimidos.

\section{Extração e caracterização do paracetamol}

Em função da escassez de relatos sobre a extração do PFA de comprimidos de paracetamol como tema de aula experimental, e dos dois exemplos conhecidos serem restritos a medicamentos de

Tabela 1. Massas dos comprimidos contendo paracetamol por categoria de medicamento e grupo de estudo

\begin{tabular}{|c|c|c|c|c|c|c|c|c|}
\hline \multicolumn{9}{|c|}{ Massas dos comprimidos (g) } \\
\hline \multirow{3}{*}{$\begin{array}{c}\text { Grupo } \\
\text { Tipo } \\
\text { Análise }\end{array}$} & \multicolumn{2}{|c|}{ UFBA } & \multicolumn{4}{|c|}{ UNEB } & \multicolumn{2}{|c|}{ Profissional } \\
\hline & \multirow{2}{*}{$\frac{\text { Genérico }}{2009 / 750^{\mathrm{a}}}$} & \multirow{2}{*}{$\frac{\text { Referência }}{2009 / 500}$} & \multicolumn{2}{|c|}{ Genérico } & \multicolumn{2}{|c|}{ Referência } & \multirow{2}{*}{$\begin{array}{l}\text { Genérico } \\
2018 / 750\end{array}$} & \multirow{2}{*}{$\begin{array}{c}\text { Referência } \\
2018 / 750\end{array}$} \\
\hline & & & $2018 / 500^{b}$ & $2019 / 750$ & $2018 / 750$ & $2019 / 750$ & & \\
\hline 1. & 0,8350 & 0,6131 & 0,608 & 0,8195 & 0,8302 & 0,8201 & 0,8311 & 0,8203 \\
\hline 2. & 0,8354 & 0,6145 & 0,610 & 0,8199 & 0,8322 & 0,8258 & 0,8342 & 0,8208 \\
\hline 3. & 0,8410 & 0,6188 & 0,610 & 0,8202 & 0,8323 & 0,8262 & 0,8347 & 0,8233 \\
\hline 4. & 0,8423 & 0,6203 & 0,610 & 0,8216 & 0,8338 & 0,8263 & 0,8353 & 0,8271 \\
\hline 5. & 0,8429 & 0,6210 & 0,611 & 0,8226 & 0,8344 & 0,8265 & 0,8355 & 0,8279 \\
\hline 6. & 0,8444 & 0,6213 & 0,611 & 0,8242 & 0,8348 & 0,8276 & 0,8364 & 0,8280 \\
\hline 70 & 0,8452 & 0,6231 & 0,613 & 0,8259 & 0,8354 & 0,8285 & 0,8367 & 0,8280 \\
\hline 8. & 0,8475 & 0,6232 & 0,613 & 0,8284 & 0,8358 & 0,8292 & 0,8373 & 0,8281 \\
\hline 9. & 0,8503 & 0,6239 & 0,614 & 0,8303 & 0,8358 & 0,8300 & 0,8377 & 0,8282 \\
\hline 10. & 0,8508 & 0,6240 & 0,616 & 0,8315 & 0,8369 & 0,8322 & 0,8379 & 0,8285 \\
\hline 11. & 0,8510 & 0,6240 & 0,616 & 0,8318 & 0,8374 & 0,8328 & 0,8385 & 0,8315 \\
\hline 12. & 0,8530 & 0,6244 & 0,616 & 0,8328 & 0,8390 & 0,8339 & 0,8386 & 0,8318 \\
\hline 13. & 0,8532 & 0,6246 & 0,617 & 0,8344 & 0,8391 & 0,8341 & 0,8390 & 0,8320 \\
\hline 14. & 0,8534 & 0,6247 & 0,617 & 0,8348 & 0,8394 & 0,8347 & 0,8399 & 0,8322 \\
\hline 15. & 0,8543 & 0,6256 & 0,618 & 0,8372 & 0,8404 & 0,8348 & 0,8404 & 0,8346 \\
\hline 16. & 0,8555 & 0,6263 & 0,619 & 0,8373 & 0,8405 & 0,8355 & 0,8407 & 0,8366 \\
\hline 17. & 0,8573 & 0,6266 & 0,620 & 0,8380 & 0,8406 & 0,8356 & 0,8414 & 0,8368 \\
\hline 18. & 0,8594 & 0,6268 & 0,620 & 0,8417 & 0,8416 & 0,8358 & 0,8417 & 0,8377 \\
\hline 19. & 0,8658 & 0,6275 & 0,621 & 0,8462 & 0,8418 & 0,8365 & 0,8436 & 0,8394 \\
\hline 20. & 0,8665 & 0,6275 & 0,630 & 0,8475 & 0,8421 & 0,8418 & 0,8440 & 0,8449 \\
\hline Mediana $^{\mathrm{c}}$ & 0,8502 & 0,6231 & 0,616 & 0,8316 & 0,8372 & 0,8325 & 0,8382 & 0,8300 \\
\hline Média $^{c}$ & 0,8509 & 0,6240 & 0,615 & 0,8313 & 0,8372 & 0,8314 & 0,8382 & 0,8309 \\
\hline Desvio médio ${ }^{c}$ & 0,0068 & 0,0029 & 0,004 & 0,0069 & 0,0030 & 0,0042 & 0,0025 & 0,0049 \\
\hline Desvio padrão $^{c}$ & 0,0087 & 0,0040 & 0,009 & 0,0085 & 0,0035 & 0,0051 & 0,0033 & 0,0062 \\
\hline Média - 5\% & 0,8084 & 0,5928 & 0,584 & 0,7897 & 0,7953 & 0,7898 & 0,7963 & 0,7894 \\
\hline Média $+5 \%{ }^{\mathrm{d}}$ & 0,8934 & 0,6552 & 0,646 & 0,8729 & 0,8791 & 0,8730 & 0,8801 & 0,8724 \\
\hline
\end{tabular}

a: ano de execução do experimento/massa de paracetamol indicada na caixa do medicamento. b: foi empregada balança semianalítica. c: parâmetros estatísticos obtidos através do Excel $^{\oplus}$. d: valores mínimo e máximo permitidos para uma amostra de 20 comprimidos segundo a Farmacopeia Brasileira. ${ }^{4}$ 
referência, foi realizada a extração sólido-líquido de comprimidos dos dois tipos de medicamentos, simplificando-se o procedimento previamente descrito. ${ }^{5}$ Outro aspecto que justifica revisitar a extração do paracetamol é a constatação de que a massa de cada comprimido dos dois tipos de medicamentos varia dentro de uma faixa relativamente ampla, o que significa que os comprimidos de um lote, e mesmo de uma caixa, apresentam também flutuação na massa do PFA, não sendo exatamente 750 ou $500 \mathrm{mg}$ de paracetamol em cada comprimido. Quando da avaliação do teor de paracetamol extraído no único experimento de extração sólido-líquido descrito para aula, ${ }^{5}$ o teor encontrado foi $65 \%$, mas como esse valor foi calculado assumindo que há exatamente $750 \mathrm{mg}$ de paracetamol em cada comprimido (os comprimidos não foram pesados), a eficácia da extração pode estar tanto super quanto subestimada.

Uma nova abordagem foi desenvolvida para contornar a não constância da massa dos comprimidos, e também quando a indisponibilidade simultânea a comprimidos de mesma massa ( 750 ou $500 \mathrm{mg}$ ) dos dois diferentes tipos de medicamento, como foi o caso em 2009 que nos levou a empregar comprimidos de $750 \mathrm{mg}$ (genérico) e de $500 \mathrm{mg}$ (referência). Assim, são pulverizados dois comprimidos de $750 \mathrm{mg}$ ou três de $500 \mathrm{mg}$ de cada tipo de medicamento sendo pesados 1,5 g de cada pó resultante para que seja extraído. Dessa forma, mesmo empregando comprimidos de massas diferentes é possível mensurar a eficácia da extração a partir da mesma massa submetida à extração, e assim pode ser feita a comparação da solubilidade do PFA nos dois tipos de medicamentos, Tabela 2.

Para a identificação do paracetamol extraído cada aluno/equipe determinou temperatura de fusão do sólido obtido (cujos valores ficaram entre $167-169^{\circ} \mathrm{C}$ ) e obteve os espectros na região do infravermelho, confirmando os grupos funcionais e o padrão de substituição do anel aromático. ${ }^{20}$ Foi verificado que todos os dados estão em concordância com os descritos na literatura. ${ }^{5,6}$

É importante destacar que o procedimento aqui modificado não tem por intenção promover a extração exaustiva do PFA da massa dos comprimidos pulverizados, mas sim submeter uma mesma quantidade de massa de comprimidos dos dois tipos de medicamentos ao mesmo método de extração e assim poder comparar a eficácia da extração.
Esse fato é importante, pois, padronizado o procedimento, a massa do PFA extraída pode ser usada como um potencial indicativo da absorção e/ou liberação do fármaco num meio lipofílico, aqui representado pelo solvente extrator (acetato de etila), de quanto maior a massa solúvel no solvente, maior absorção/liberação de um tipo de medicamento em relação ao outro.

A percentagem de massa extraída em cada conjunto de comprimidos foi determinada em triplicata, Tabela 2. Os valores para os dois tipos de medicamentos foram próximos para os experimentos executados num mesmo ano, mas os valores encontrados em 2009 (genérico $59 \pm 17 \%$, de referência $56 \pm 6 \%$ ) foram o dobro dos obtidos dez anos depois. A razão para esse fato não está clara, mas pode estar relacionado à variação da granulometria do pó resultante da pulverização do comprimido, uma vez que quanto menor o tamanho das partículas, maior a superfície do sólido de contato e mais eficaz é a extração sólido-líquido para o mesmo volume do solvente extrator e tempo de contato solvente-sólido. Todavia, como houve reprodutibilidade do percentual de massa extraída para as amostras dos anos 2018/2019 (genérico $29 \pm 1 \% / 32 \pm 1 \%$, de referência $32 \pm 1 \% / 32 \pm 0 \%$ ) entre grupos de alunos distintos e profissionais experientes (para 2018: genérico $29 \pm 1 \%$, de referência $28 \pm 1 \%$ ), a discrepância desse parâmetro em relação às amostras de 2009 pode estar mais relacionado com eventual mudança de formulação farmacêutica ou com a ocorrência de polimorfismo do paracetamol ${ }^{21}$ do que com a não acurácia dos alunos na execução do experimento. São conhecidas cinco diferentes formas de polimorfos do paracetamol (denominadas formas I a V), sendo três completamente caracterizadas e, por apresentam propriedades físico-químicas diferentes, possuem solubilidade e velocidade de dissolução diferentes num mesmo solvente. A forma I é a mais estável e comum nas formulações farmacêuticas, mas requer a adição de excipientes para a obtenção do comprimido, e a velocidade de dissolução depende também do tipo do excipiente empregado. ${ }^{21}$ Qualquer que seja a origem da discrepância, não há diferença estatisticamente significativa no percentual de paracetamol extraído do par de medicamentos genérico e de referência de um mesmo ano, para todos as amostras investigadas empregando o procedimento aqui descrito.

Tabela 2. Extração do paracetamol dos comprimidos por categoria de medicamento e grupo de estudo

\begin{tabular}{|c|c|c|c|c|c|c|c|c|}
\hline \multicolumn{9}{|c|}{ Massas de paracetamol (g) } \\
\hline \multirow{2}{*}{$\begin{array}{c}\text { Grupo } \\
\text { Tipo }\end{array}$} & \multicolumn{2}{|l|}{ UFBA } & \multicolumn{4}{|c|}{ UNEB } & \multicolumn{2}{|l|}{ Profissional } \\
\hline & Genérico & Referência & \multicolumn{2}{|c|}{ Genérico } & \multicolumn{2}{|c|}{ Referência } & \multirow{2}{*}{$\begin{array}{l}\text { Genérico } \\
2018 / 750\end{array}$} & \multirow{2}{*}{$\begin{array}{c}\text { Referência } \\
2018 / 750\end{array}$} \\
\hline Análise & $2009 / 750^{a}$ & $2009 / 500$ & $2018 / 500$ & $2019 / 750$ & $2018 / 750$ & $2019 / 750$ & & \\
\hline 1 & 1,5066 & 1,5505 & 1,5013 & 1,5038 & 1.5008 & 1,5078 & 1,5188 & 1,5118 \\
\hline 1 & 0,6609 & 0,8332 & 0,4565 & 0,4853 & 0,4954 & 0,4755 & 0,4361 & 0,4364 \\
\hline 2 & 1,5043 & 1,5170 & 1,5053 & 1,5016 & 1.5002 & 1,5110 & 1,5112 & 1,5149 \\
\hline 2 & 0,8596 & 0,9628 & 0,4470 & 0,4975 & 0.5145 & 0,4927 & 0,4478 & 0,4171 \\
\hline 3 & 1,5072 & 1,5008 & 1,5018 & 1,5009 & 1.5011 & 1,5110 & 1,5141 & 1,5124 \\
\hline 3 & 1,1605 & 0,7726 & 0,4259 & 0,4703 & 0.4378 & 0,4810 & 0,4184 & 0,4209 \\
\hline \multicolumn{9}{|l|}{ Massa Extraida (\%) } \\
\hline 1 & 44 & 55 & 30 & 32 & 33 & 32 & 29 & 29 \\
\hline 2 & 57 & 63 & 30 & 33 & 34 & 32 & 30 & 28 \\
\hline 3 & 77 & 51 & 28 & 31 & 29 & 32 & 28 & 28 \\
\hline Mediana $^{c}$ & 57 & 55 & 30 & 32 & 33 & 32 & 29 & 28 \\
\hline Média & 59 & 56 & 29 & 32 & 32 & 32 & 29 & 28 \\
\hline Desvio médio & 12 & 4 & 1 & 1 & 2 & 0 & 1 & 0 \\
\hline Desvio padrão & 17 & 6 & 1 & 1 & 3 & 0 & 1 & 1 \\
\hline
\end{tabular}

a: para cada Análise, o primeiro número é a massa do comprimido pulverizado empregado na extração e o número imediatamente abaixo a este é a massa de paracetamol extraído. b: foi empregada balança semianalítica. c: parâmetros estatísticos referentes à massa extraída, obtidos através do Excel ${ }^{\oplus}$. 
Em função da redução da massa extraída dos comprimidos dos anos 2018-9 uma segunda abordagem foi desenvolvida, e para que se tenha mais opções de análise nos casos de laboratório de ensino com melhor infraestrutura e/ou maior duração de aula, como os que envolvem a execução de projetos simples. Para isso, foi executada a extração sólido-líquido dos comprimidos de 2018 empregando o aparelho Soxhlet. Cerca de 1,5 gramas de cada tipo de medicamento foi extraída com $100 \mathrm{~mL}$ de acetato de etila, e o tempo máximo de extração foi fixado em 1 hora de forma a permitir a execução no contexto de aula mais amplo, como mencionado. $\mathrm{O}$ volume do solvente extrator foi maior em função do volume morto que fica no balão sob aquecimento, mas o volume de contato com o cartucho contendo o sólido foi aproximadamente o mesmo da extração clássica. Nessa condição, a percentagem de massa extraída do medicamento de referência foi $80 \%$ e do genérico $85 \%$. Os espectros na região do infravermelho dos comprimidos pulverizados, do paracetamol extraído e do pó residual da extração são praticamente idênticos entre si (Figuras $2 \mathrm{~S}$ a $6 \mathrm{~S}$ ) e entre os dois medicamentos, e a única diferença observada foi na temperatura de fusão do pó residual da extração, Tabela $1 \mathrm{~S}$.

\section{CONCLUSÃO}

A aula experimental desenvolvida se mostrou eficiente para o ensino da técnica de extração sólido-líquido de compostos orgânicos, exemplificado com o paracetamol, e proporcionou o treinamento na caracterização de substância orgânica por meio da determinação da temperatura de fusão e da obtenção e interpretação do espectro na região do infravermelho, questões importantes no ensino experimental de química para diversos profissionais. Todavia, a aula não é restrita a esses aspectos, pois explora também parâmetros inerentes ao controle de qualidade da fabricação de medicamentos, como a dispersão das massas dos comprimidos e o tratamento estatísticos desses dados. Como os procedimentos são simples, utilizam matéria prima facilmente adquirida e empregam vidrarias e equipamentos típicos de laboratórios de ensino, permitem a execução em diferentes contextos de infraestrutura. Em função do caráter modular dos dois ciclos do experimento, a estratégia desenvolvida pode ser adotada em programas de Química e Farmácia sob outros enfoques.

Outros aspectos subjacentes à atividade didática desenvolvida são tão importantes quanto o aprendizado da técnica de extração pois, nos três grupos de estudantes que realizaram a aula, a percepção majoritária inicial era que o medicamento genérico seria supostamente de qualidade inferior ao de referência por, possivelmente, possuir menor quantidade de princípio ativo e/ou não ser tão eficaz quanto o mesmo. Apesar de não avaliar todos os aspectos inerentes ao tema, a comparação entre medicamentos genérico e de referência como elemento motivacional revela aos estudantes que, sob a ótica das medidas realizadas das variáveis investigadas no laboratório de ensino, os dois tipos de medicamentos são comparáveis. Ficam alertados que a avaliação científica é a fonte das decisões racionais e que a Ciência é o guia da formação de opinião técnica, principalmente para as gerações de profissionais que estão sendo formadas no ambiente das fake news.

\section{PARTE EXPERIMENTAL}

Os medicamentos contendo paracetamol foram adquiridos em estabelecimentos comerciais farmacêuticos localizados na cidade de Salvador - BA, todos dentro do prazo de validade dos respectivos lotes, e tanto o de referência quanto o genérico de comprimidos de $750 \mathrm{mg}$ e $500 \mathrm{mg}$. Os pontos de fusão foram determinados em um aparelho de placa aquecida Microquímica MQAPF 301 e não foram corrigidos. Os espectros na região do infravermelho foram obtidos na forma de disco de $\mathrm{KBr}$ em um aparelho SHIMADZU IR Affinity-1.

\section{Aferição da massa dos comprimidos dos medicamentos de} paracetamol

Desembalar os comprimidos dos medicamentos genéricos e de referência acondicionando-os em recipientes distintos, tomando o cuidado de não tocar nos mesmos, sempre manipulando-os com o auxílio de uma pinça metálica. Cada equipe deve pesar ao menos três comprimidos numa balança analítica, anotar as massas e prosseguir à extração como descrito abaixo.

\section{Extração do paracetamol (adaptado da referência 5)}

Usar os comprimidos do medicamento genérico ou referência previamente pesados e executar o experimento a seguir. Em um almofariz, triturar com um pistilo dois comprimidos de $750 \mathrm{mg}$ de paracetamol (ou três de $500 \mathrm{mg}$ ) até obter um pó fino homogêneo. Pesar aproximadamente 1,5 g deste pó, anotar a massa e transferir para um erlenmeyer de $125 \mathrm{~mL}$. Adicionar $40 \mathrm{~mL}$ de acetato de etila e agitar a suspensão vigorosamente com movimentos circulares. Agitar ocasionalmente por mais 5 min e em seguida filtrar para um béquer de $100 \mathrm{~mL}$ previamente pesado. Lavar o sólido residual do erlenmeyer com mais $10 \mathrm{~mL}$ de acetato de etila. Colocar o béquer com o líquido em uma placa de aquecimento previamente aquecida a $60{ }^{\circ} \mathrm{C}$, evaporar o líquido até quase secura e deixar secar ao ar por uma semana. Após esse tempo, pesar o béquer com o sólido branco para determinar o teor de massa extraída. Com uma espátula raspar o sólido das paredes do recipiente e homogeneizar, determinar o ponto de fusão e obter o espectro na região do infravermelho.

Alternativamente, na etapa de filtração é possível substituir o béquer por um balão previamente pesado para eliminar o solvente em um evaporador rotatório, se a infraestrutura necessária estiver disponível.

\section{Extração do paracetamol empregando o aparelho de Soxhlet}

Utilizando comprimidos do medicamento genérico ou referência previamente pesados, executar o procedimento descrito a seguir. Em um almofariz, triturar com um pistilo dois comprimidos de $750 \mathrm{mg}$ de paracetamol até obter um pó fino homogêneo. Pesar aproximadamente 1,5 g do material pulverizado, anotar a massa e transferir para um cartucho de papel de filtro. Introduzir o cartucho contendo o material pulverizado no extrator de Soxhlet já conectado a um balão de fundo chato de $200 \mathrm{~mL}$ e colocar em uma placa de aquecimento. Adicionar suavemente $100 \mathrm{~mL}$ de acetato de etila no centro da cavidade do Soxhlet de forma a ter uma primeira extração a frio. Conectar o condensador e aquecer o sistema de forma a ter refluxo suave, gota a gota. Interromper o aquecimento após 40 minutos ou quando completados três ciclos de extração a quente (o que ocorrer primeiro). Por meio de uma pinça remover cuidadosamente o cartucho da cavidade do Soxhlet, transferir o solvente que remanescente para um balão de fundo redondo previamente pesado e transferir para este o solvente contido no balão de fundo chato. Eliminar o solvente num evaporador rotatório e determinar a massa residual. Obtém-se um sólido branco cristalino. Caracterizar como descrito na extração clássica.

\section{MATERIAL SUPLEMENTAR}

Fotos do procedimento da extração com Soxhlet, tabela com temperaturas de fusão e espectros na região do infravermelho do paracetamol extraído, do sólido residual não extraído e do pó obtido da pulverização dos comprimidos estão disponíveis em http:// quimicanova.sbq.org.br, na forma de arquivo PDF, com acesso livre.

\section{AGRADECIMENTOS}

Os autores agradecem o suporte financeiro do Conselho Nacional de Desenvolvimento Científico e Tecnológico - CNPq, da 
Fundação de Amparo à Pesquisa do Estado da Bahia - FAPESB e da Coordenação de Aperfeiçoamento de Pessoal de Nível Superior Brasil (CAPES) - Código de Financiamento 001. Ao programa PICIN/ UNEB pela bolsa de iniciação científica de O.M.B.

\section{REFERÊNCIAS}

1. Maluta, J. R.; Quim. Nova 2014, 37, 1244; Pazinato, M. S.; Braibante, H. T. S.; Braibante, M. E. F.; Trevisan, M. C.; Silva, G. S.; Quim. Nova Esc. 2012, 34, 21; Brown, M. E.; Cosser, R. C.; Davies-Coleman, M. T.; Kaye, P. T.; Klein, R.; Lamprecht, E.; Lobb, K.; Nyokong, T.; Sewry, J. D.; Tshentu, Z. R.; Zeyde, T. v. d.; Watkins, G. M.; J. Chem. Educ. 2010, 87,500 .

2. Bodek, M.; Burch, M.; Cannon, J.; Finneran, D.; Geveke, K.; Sinkinson, H.; Smith, W.; Tierney J.; J. Chem. Educ. 2020, 97, 574; da Rocha, T. R.; Braibante, H. T. S. Em Temáticas para o Ensino de Química: Contribuições com Atividades Experimentais; Braibante, M. E. F.; Braibante, H. T. S., eds.; Editora CVR: Curitiba, 2019, cap 10; Josepshon, P.; Nykvist, V.; Qasim, W.; Blomkvist, B.; Diner, P. J.; J. Chem. Educ. 2019, 96, 1389; Rennie, T.; Eelu, H.; Lusepani, M.; Keendjele, T.; Julka, E.; Hunter, C.; J. Chem. Educ. 2019, 96, 1096; Silva, R. S.; Borges, E. M.; J. Chem. Educ. 2019, 96, 1519; Gois, L. C.; de Borba, W. G.; da Silva, J. G.; Rev. Virtual Quim. 2019, 11, 958; Goes Junior, E. J. A.; Roeder, J. S.; Oliveira, K. B. L.; Ferreira, M. P.; da Silva, J. G.; Quim. Nova 2019, 42, 99; Tavares, M. T. T.; Primi, M. C.; Carvalho, C. F.; Polli, M. C.; Parise-Filho, R.; Quim. Nova 2016, 39, 502; Gonsalves, A. A.; Araújo, C. R. M.; Filho, C. A. L.; Medeiros, F. S.; Quim. Nova 2013, 36, 1236; Arruda, I. E.; Macedo, B. V. S.; Macedo, J. C.; Campos, W. R. A.; Araújo, C. R. M.; Gonsalves, A. A.; Quim. Nova 2020, 43, 642; de Souza, A. M.; Breitkreitz, M. C.; Filgueiras, P. R.; Rohwedder, J. J. R.; Poppi, R. J.; Quim. Nova 2013, 36, 1057; Leslie, R.; Leeb, E.; Smith, R. B.; J. Chem. Educ. 2012, 89, 144; Pereira, A. V.; Garabeli, A. A.; Schunemann, G. D.; Borck, P. C.; Quim. Nova 2011, 34, 1656; Suarez, W. T.; Ferreira, L. H.; Fatibello-Filho, O.; Quim. Nova Esc. 2007, 25, 36; Eleotério, I. C.; Kiill, K. B.; de Sene, J. J.; Ferreira, L. H.; Hartwig, D. R.; Quim. Nova Esc. 2007, 26, 37; Haddad, P.; Rasmussen, M.; J. Chem. Educ. 1976, 53, 731.

3. Dias, L. C.; Dessoy, M. A.; Barreiro, E. J.; Caracterização do conteúdo das cápsulas de fosfoetanolamina (FOS) para o MCTI. Disponível em: https://www.mctic.gov.br/mctic/export/sites/institucional/ciencia/ SEPED/Saude/fosfoetanolamina/arquivos/Relatorio-Completo-deSintese-e-Caracterizacao.pdf, acessada em Julho 2020; Oliveira, A. G.; Silveira, D. Infarma 2016, 28, 3.

4. Farmacopeia Brasileira. $6^{a}$ ed. Agência Nacional de Vigilância Sanitária: Brasília (DF), 2019, 1, 61 .
5. Baptistella, L. H. B.; Glacomini, R. A.; Imamura, P. M.; Quim. Nova 2003, 26, 284.

6. Revell, K. D.; J. Chem. Educ. 2011, 88, 1413.

7. Zivkovic, A.; Bandolik, J. J.; Skerhut, A. J.; Coesfeld, C.; Prascevic, M.; Zivkovic, L.; Stark, H.; J. Chem. Educ. 2017, 94, 121; Araújo, C. R. M.; Filho, C. A. L.; Santos, V. L. A.; Maia, G. L.; Gonsalves, A. A.; Quim. Nova 2015, 38, 868; Hulien, M. L.; Lekse, J. W.; Rosmus, K. A.; Devlin, K. P.; Glenn, J. R.; Wisneski, S. D.; Wildfong, P.; Lake, C. H.; MacNeil, J. H.; Aitken, J. A.; J. Chem. Educ. 2015, 92, 2152; Parmeggiani, F.; J. Chem. Educ. 2014, 91, 692; Myrick, M. L; Baranowski, M.; J. Chem. Educ. 2010, 87, 842; Mott, J. R.; Munson, P. J.; Kreuter, R. A.; Chohan, B. S.; Sykes D. G.; J. Chem. Educ. 2014, 91, 1028; Rybolt, T. R.; Burrell, D. E.; Shults, J. M.; Kelley A. K.; J. Chem. Educ. 1988, 65, 1009; Yarnelle, M. K.; West, K. J.; J. Chem. Educ. 1988, 66, 601.

8. Cunha, S.; Rodrigues, M. C.; Mattos, R. R.; Teixeira, L. S. G.; Santos, A. O.; Santos, E. V.; Souza, R. S.; Andrade, G. S.; De Paula, R.; Jesus, D. S.; Quim. Nova 2018, 41, 691; Cunha, S.; Fontes, T.; Araújo, D. M., Riatto, V. B.; Quim. Nova 2018, 41, 116; Cunha, S.; Matos, J. S.; Quim. Nova 2017, 40, 1253; Cunha, S.; Costa, O. B. S.; Santana, L. L. B.; Lopes, W. A.; Quim. Nova 2015, 38, 874; Cunha, S.; Lustosa, D. M.; Conceição, N. D.; Fascio, M.; Magalhães, V.; Quim. Nova 2012, 35, 638.

9. Rosenberg, G.; Fonseca, M. G. D.; D'avila, L. A.; Econ. Soc. 2010, 19, 107.

10. Brasil. Lei $\mathrm{N}^{\circ} 6.360$ de 24 de setembro de 1976.

11. Coutinho, M. E.P.; Moura, M. C. L.; Araújo, V. L. L.; Cavalcanti, G. L.; Amaral, M. P. M.; Rev. Uningá 2018, 55, 62.

12. Brasil. Lei $\mathrm{N}^{\circ} 9.787$, de 10 de fevereiro de 1999.

13. Bertoldi, A. D.; Arrais, P. S. D.; Tavares, N. U. L.; Ramos, L. R.; Luiza, V. L.; Mengue, S. S.; Dal-Pizzo, T.S.; Farias, M. R; Oliveira, M. A. Rev. Saude Publica 2016, 50, 1.

14. Silva, N. C. S.; Rocha, L. C.; Unica Cadernos Academicos 2016, 3, 2.

15. Brasil. Agência Nacional de Vigilância Sanitária, Resolução RDC n ${ }^{\circ}$ 135, de 29 de maio de 2003.

16. BRASIL. Agência Nacional de Vigilância Sanitária. Resolução RDC 310, de 19 de agosto de 2019.

17. Oliveira, A. C. A. X.; Paumgartten, F. J. R.; Cienc. Saude Coletiva 2017, 22, 2549

18. Bate, R.; Mathur, A.; Lever, H. M.; Thakur, D.; Graedon, J.; Cooperman, T.; Mason, P.; Fox, E. R.; Trends Pharmacol. Sci. 2016, 37, 184

19. Araújo, L. U.; Albuquerque, K. T.; Kato, K. C.; Silveira, G. S.; Maciel N. R.; Spósito; P. A.; Barcelo, N. M. S.; Souza, J.; Bueno, M.; Storpirtis, S.; Rev. Panam. Salud Publica 2010, 28, 480.

20. Lopes, W. A.; Fascio M.; Quim. Nova 2004, 27, 670.

21. Cruz, P. C.; Rocha, F. A.; Ferreira, A. M.; Org. Process Res. Dev. 2019, 23, 2592 . 